\title{
The Sommerfeld Half-Plane Problem Revisited I: The Solution of a Pair of Coupled Wiener-Hopf Integral Equations*)
}

\author{
A. E. Heins, Ann Arbor \\ Communicated by E. Meister
}

\begin{abstract}
A study and the solution of an extension of the classical Sommerfeld half-plane problem which leads to a pair of integral equations of the Wiener-Hopf type is given. The method of solution is function theoretic in character and employs a combination of the ideas of Wiener and Hopf and Carleman.
\end{abstract}

\section{Introduction}

It is now more than eighty years since $A$. Sommerfeld [10] produced the solution of the first problem in diffraction theory. In a paper entitled "Zur Theorie der Diffraktion-Theorie" we see the first solution of the problem which is now known as the Sommerfeld half-plane problem. This problem dealt with the diffraction of a plane wave by a "soft" or "hard" half-plane. That is, it dealt mathematically with the study of the solutions of the two dimensional wave equation which obey Dirichlet boundary conditions ("soft surface") on both sides of the half-plane or Neumann boundary conditions ("hard surface") on both sides of the half-plane. It is necessary to describe the conditions at infinity in order to provide a physically and mathematically meaningful problem, and we shall discuss this point in Section II of this paper. Although the results of Sommerfeld provided insights into the nature of some diffractive elements, his mathematical methods do not lend themselves to further studies.

In 1943, it was noted by J. Schwinger that a much wider class of geometries could be considered by formulating such problems as integral equations of the Wiener-Hopf type. Such problems were studied by his co-workers J. F. Carlson [3], A. E. Heins [3] and H. Levine [7]. The problems considered by them were formulated as a single integral equation of the Wiener-Hopf type and thus fall under the general ideas considered in [8]. It should be mentioned that there

-) Presented at a Conference in Diffraction and Scattering Theory held at the Technische Hochschule Darmstadt on May 29, 1980. 
were several technical details which had to be overcome which dealt in part with the formulation of the integral equations and in part with some questions in complex analysis.

To date, there has been very little progress in the solution of systems of such integral equations. Naively, one would hope to be able to factor a matrix of analytic functions in much the same fashion which can be done for the single analytic function in Wiener-Hopf Theory [5]. Unfortunately, such is not the case since, at present, we do not have the tools to do this for many of these integral equations which arise in the physical sciences. We should note here that the analytic structure of these matrices are not as simple as those considered by $\mathrm{H}$. Bart, I. Gohberg and M. A. Kaashoek [1]. A simple example of a system was provided by the present author in 1950 [5] but it relies on the periodicity of the given geometric structure - a wave guide with $n$ equally spaced, semi-infinite plates. This problem depends basically on a single analytic function. In 1975, A. D. Rawlins [9] reconsidered the half-plane problem of Sommerfeld with the following change in boundary conditions. On one side of the half-plane, the Dirichlet boundary condition is to be satisfied while on the other side the Neumann boundary condition is to be satisfied. This gives rise to a pair of coupled integral equations of the Wiener-Hopf type for which, in Rawlins' own words, an "ad-hoc method" of solution is provided. Our goal is to demonstrate that we can provide a constructive method for solving this system which depends in part on the original ideas of Wiener and Hopf and in part on Carleman's ideas on singular integral equations. Both of these ideas, in turn, have their origin in the theory of analytic functions. We remark that we do not "factor" the matrix involved. We rely heavily on the particular analytic structure of the Fourier transforms of the various kernels involved and at present cannot supply any further examples.

There is also the work of Hurd [6] who studies the same geometric structure as Rawlins but now invokes different impedance boundary conditions on each face of the half-plane. This problem also leads to a coupled pair of WienerHopf integral equations. Hurd provides a solution to this problem which he claims had been obtained by factoring the matrix of the Fourier transforms of the various kernels in the Wiener-Hopf system. This problem is more difficult than the one of Rawlins and takes into account (in a narrow sense) the physical nature of the half-plane.

\section{The Formulation of the Problem}

We recall that for a plane wave incident upon a half-plane $x>0, y=0$

$$
\begin{aligned}
\phi(x, y)= & \phi_{\text {inc }}(x, y) \\
& \left.+\frac{i}{4} \int_{0}^{\infty} H_{0}^{(1)}\left[k \sqrt{\left(x-x^{\prime}\right)^{2}+y^{2}}\right] \phi_{y^{\prime}}\left(x^{\prime},-0\right)-\phi_{y^{\prime}}\left(x^{\prime},+0\right)\right] \mathrm{d} x^{\prime} \\
& -\frac{i}{4} \int_{0}^{\infty} \frac{\partial H_{0}^{(1)}}{\partial y}\left[k \sqrt{\left(x-x^{\prime}\right)^{2}+y^{2}}\right]\left[\phi\left(x^{\prime},+0\right)-\phi\left(x^{\prime},-0\right)\right] \mathrm{d} x^{\prime}
\end{aligned}
$$


is a representation of the solution of the equation

$$
\phi_{x x}+\phi_{y y}+k^{2} \phi=0
$$

which incorporates the Sommerfeld radiation condition as well as the incident wave. In the present case since we have assumed that the incident wave $\phi_{\text {inc }}(x, y)$ is a plane wave, we have $\phi_{\text {inc }}(x, y)=\exp \left[i\left(x k_{x}+y k_{y}\right)\right]$ where $k_{x}=k \cos \alpha$ and $k_{y}=k \sin \alpha, 0<\alpha<\pi$ and $\alpha$ is the angle which the propagation normal makes with respect to the positive $x$ axis. We shall assume here that $\operatorname{Im} k>0$. The notation $\phi(x,+0)$ implies that $\lim _{y \rightarrow+0} \phi(x, y)=\phi(x,+0)$ and $\frac{\partial \phi}{\partial y}(x,+0)$ implies that $\lim _{y \rightarrow+0} \frac{\partial \phi(x, y)}{\partial y}=\frac{\partial \phi(x,+0)}{\partial y}$. Similar interpretations are available for $\phi(x,-0)$, etc. The function

$$
\frac{i}{4} H_{0}^{(1)}\left[k \sqrt{\left(x-x^{\prime}\right)^{2}+\left(y-y^{\prime}\right)^{2}}\right]
$$

is a Hankel function of the first kind and is the free space Green's function for the equation (2.2) which obeys the Sommerfeld radiation condition. That is, for fixed $\left(x^{\prime}, y^{\prime}\right)$ and $r=\sqrt{\left(x^{2}+y^{2}\right)}$,

$$
\frac{\partial H_{0}^{(1)}}{\partial r}-\mathrm{i} k H_{\delta}^{(1)}=O\left(\frac{1}{r^{3 / 2}}\right), \quad r \rightarrow \infty .
$$

The particular boundary value problem which we wish to investigate deals with the situation $\phi_{y}(x,+0)=\phi(x,-0)=0$, the case which Rawlins [8] investigated several years ago. The classical cases which Sommerfeld investigated are $\phi(x,+0)=\phi(x,-0)=0$ or $\frac{\partial \phi(x,+0)}{\partial y}=\frac{\partial \phi(x,-0)}{\partial y}=0$ and lead to integral equations of the Wiener-Hopf type for a single unknown for either the discontinuity of the normal derivative of $\phi$ (in the Dirichlet case) or the discontinuity of $\phi$ (in the Neumann case). Now, in the case which Rawlins examined we are confronted with a system of Wiener-Hopf integral equations for the unknowns $\phi_{y}(x,-0)=f_{1}(x)$ and $\phi(x,+0)=f_{2}(x), x>0$. Indeed when we invoke the second boundary condition $\phi(x,-0)=0, x>0$, we obtain from (2.1)

$$
0=\exp \left(\mathrm{i} x k_{x}\right)+\frac{\mathrm{i}}{4} \int_{0}^{\infty} H_{0}^{(1)}\left[k\left|x-x^{\prime}\right|\right] f_{1}\left(x^{\prime}\right) \mathrm{d} x^{\prime}-\frac{f_{2}(x)}{2}
$$

The term $-f_{2}(x) / 2$ arises since the Hankel function $H_{0}^{(1)}\left[k \sqrt{\left(x-x^{\prime}\right)^{2}+y^{2}}\right]$ contains a term which is proportional to $\ln \sqrt{\left(x-x^{\prime}\right)^{2}+y^{2}}$ when $x \rightarrow x^{\prime}, y \rightarrow 0$. The derivative of the Hankel function therefore contains a term which is proportional to the two-dimensional Poisson kernel for a half-space, all others vanishing with $y$. It is this Poisson term which produces $-f_{2}(x) / 2$. Let us note that if we had taken the limit of (2.1) with $y \rightarrow+0$, we would have obtained for $x>0$, 


$$
f_{2}(x)=\exp \left(\mathrm{i} x k_{x}\right)+\frac{\mathrm{i}}{4} \int_{0}^{\infty} H_{0}^{(1)}\left[k\left|x-x^{\prime}\right|\right] f_{1}\left(x^{\prime}\right) \mathrm{d} x^{\prime}+\frac{f_{2}(x)}{2}
$$

which upon simplification, is still (2.3).

The second boundary condition which we have to invoke is that $\frac{\partial \phi(x,+0)}{\partial y}=0, x>0$. Then for $y \neq 0,(2.1)$ becomes

$$
\begin{aligned}
\frac{\partial \phi}{\partial y}= & \mathrm{i} k_{y} \exp \left(\mathrm{i} x k_{x}+\mathrm{i} y k_{y}\right) \\
& +\frac{\mathrm{i}}{4} \int_{0}^{\infty} \frac{\partial}{\partial y} H_{0}^{(1)}\left[k \sqrt{\left(x-x^{\prime}\right)^{2}+y^{2}}\right] f_{1}\left(x^{\prime}\right) \mathrm{d} x^{\prime} \\
& -\frac{\mathrm{i}}{4} \int_{0}^{\infty} \frac{\partial^{2}}{\partial y^{2}} H_{0}^{(1)}\left[k \sqrt{\left(x-x^{\prime}\right)^{2}+y^{2}}\right] f_{2}\left(x^{\prime}\right) \mathrm{d} x^{\prime}
\end{aligned}
$$

In view of the fact that $H_{0}^{(1)}\left[k \sqrt{\left(x-x^{\prime}\right)^{2}+y^{2}}\right]$ satisfies the two dimensional homogeneous wave equations when $y \neq 0$, (2.4) may be rewritten as

$$
\begin{aligned}
\frac{\partial \phi}{\partial y}= & \mathrm{i} k_{y} \exp \left(\mathrm{i} x k_{x}+\mathrm{i} y k_{y}\right) \\
& +\frac{\mathrm{i}}{4} \int_{0}^{\infty} \frac{\partial}{\partial y} H_{0}^{(1)}\left[k \sqrt{\left(x-x^{\prime}\right)^{2}+y^{2}}\right] f_{1}\left(x^{\prime}\right) \mathrm{d} x^{\prime} \\
& +\frac{\mathrm{i}}{4}\left(\frac{\partial^{2}}{\partial x^{2}}+k^{2}\right) \int_{0}^{\infty} H_{0}^{(1)}\left[k \sqrt{\left(x-x^{\prime}\right)^{2}+y^{2}}\right] f_{2}\left(x^{\prime}\right) \mathrm{d} x^{\prime} .
\end{aligned}
$$

When $y \rightarrow+0$ and $x>0$ we get

$$
\begin{aligned}
0= & \mathrm{i} k_{y} \exp \left(\mathrm{i} x k_{x}\right)-\frac{f_{1}(x)}{2} \\
& +\frac{\mathrm{i}}{4}\left(\frac{\partial^{2}}{\partial x^{2}}+k^{2}\right) \int_{0}^{\infty} H_{0}^{(1)}\left[k\left|x-x^{\prime}\right|\right] f_{2}\left(x^{\prime}\right) \mathrm{d} x^{\prime} .
\end{aligned}
$$

This limit has to be verified after $f_{1}(x)$ and $f_{2}(x)$ are determined. It is clear that we cannot bring the differential operator under the integral sign since the integral would then diverge. What we shall ultimately show is that the integral in (2.6) converges absolutely and indeed is of the order of $|x|^{5 / 4}$ when $x \rightarrow 0$. We further will show that the integral is of the order $\exp (i k|x|) /|x|^{1 / 2}$ when $|x| \rightarrow \infty$. In view of the fact that $k$ has a positive imaginary part, we can justify our position that we can apply the Fourier integral theorem in the complex domain to this integral and eliminate the terms which arise from integration by parts. We observe that (2.3) and (2.6) form a coupled pair of integral equations of the Wiener-Hopf type (actually differential-integral equations). That is, the limits of integration are on the line $y=0,0 \leqslant x<\infty$ and the kernels are of the convolution type. The kernels possess the required behavior at infinity for the application of the Fourier integral theorem. 
III The Fourier Transform of Equations (2.3) and (2.6)

We recall that we do not know $\phi(x, 0)$ when $x<0$. We therefore rewrite (2.3) as follows, in much the same fashion which we do when we have a single Wiener-Hopf integral equation. That is,

$$
F_{1}(x)=\phi_{0}(x, 0)+\frac{\mathrm{i}}{4} \int_{0}^{\infty} f_{1}\left(x^{\prime}\right) H_{0}^{(1)}\left[k\left|x-x^{\prime}\right|\right] \mathrm{d} x^{\prime}-\frac{f_{2}(x)}{2},
$$

where now

$$
-\infty<x<\infty
$$

$$
\begin{aligned}
\phi_{0}(x, 0) & =\phi_{\text {inc }}(x, 0), & & x>0 \\
& =0, & & x<0 \\
f_{1}(x) & =0, & & x<0 \\
f_{2}(x) & =0, & & x<0
\end{aligned}
$$

and $\quad F_{1}(x) \equiv \phi(x,-0) \equiv 0, \quad x>0$.

Now we observe that

$$
F_{1}(x)=C \int_{0}^{\infty} \frac{\exp \left[i k\left(x^{\prime}-x\right)\right] f_{1}\left(x^{\prime}\right) \mathrm{d} x^{\prime}}{\sqrt{x^{\prime}-x}}
$$

when $x \rightarrow-\infty$ and where $C$ is a constant. This in turn is numerically less than

$$
|C| \int_{0}^{\infty} \frac{\exp \left[-k_{2}\left(x^{\prime}-x\right)\right]\left|f_{1}\left(x^{\prime}\right)\right| \mathrm{d} x^{\prime}}{\sqrt{x^{\prime}+\gamma}}
$$

where $\gamma$ is some positive constant and $k_{2}=\operatorname{Im} k>0$. We have therefore that $F_{1}(x)=O\left(\exp k_{2} x\right), x \rightarrow-\infty$. Furthermore, we assume (and shall verify later) that $F_{1}(x)$ is integrable in the neighborhood of the origin. We have then that the Fourier transform of $F_{1}(x)$ is

$$
\int_{-\infty}^{\infty} F_{1}(x) \exp (-\mathrm{i} w x) \mathrm{d} x=\int_{-\infty}^{0} F_{1}(x) \exp (-\mathrm{i} w x) \mathrm{d} x=\hat{F}_{1}(w)
$$

is analytic in the upper half plane $\operatorname{Im} w>-\operatorname{Im} k=-k_{2}$. The Fourier transform of $\phi_{0}(x)$ is

$$
\int_{-\infty}^{\infty} \phi_{0}(x) \exp (-\mathrm{i} w x) \mathrm{d} x=\int_{0}^{\infty} \phi_{0}(x) \exp (-\mathrm{i} w x) \mathrm{d} x=\frac{1}{\mathrm{i}\left(w-k_{x}\right)}
$$

and this is analytic in the lower half plane $\operatorname{Im} w<k_{2} \cos \alpha$. The Fourier transform of the Hankel function in (2.3) is

$$
\frac{\mathrm{i}}{4} \int_{-\infty}^{\infty} H_{0}^{(1)}[k|x|] \exp (-\mathrm{i} w x) \mathrm{d} x=\frac{\mathrm{i}}{2 \sqrt{k^{2}-w^{2}}}
$$


where $\sqrt{k^{2}-w^{2}}$ is chosen to be equal to $k=|k| \exp (\mathrm{i} \arg k), 0<\arg k<\pi / 2$ for $w=0$ and this is analytic in the strip $-k_{2}<\operatorname{Im} w<k_{2}$.

Now we shall also anticipate that $f_{1}(x)$ is integrable in the neighborhood of the origin. Actually, an edge condition calculation would tell us that $f_{1}(x)=$ $O\left(x^{-3 / 4}\right), x \rightarrow 0+. f_{1}(x)$ is also of the order $\exp (i x k \cos \alpha)$ when $x \rightarrow \infty$. The Fourier transform of $f_{1}(x)$ is therefore analytic in the lower half plane $\operatorname{Im} w<$ $k_{2} \cos \alpha$. Similarly, the edge condition tells us that $f_{2}(x)=O\left(x^{1 / 4}\right)$ when $x \rightarrow$ $0+$. In view of the fact there is no plane wave contribution for $\phi(x,-0)$ the Fourier transform of $\phi(x,-0)=f_{2}(x)$ is analytic in the lower half plane $\operatorname{Im} w<$ $\operatorname{Im} k$. Subject then to later verification

$$
\hat{F}_{1}(w)=\frac{1}{\mathrm{i}\left(w-k_{x}\right)}+\frac{\mathrm{i} \hat{f}_{1}(w)}{2 \sqrt{k^{2}-w^{2}}}-\frac{\hat{f}_{2}(w)}{2}
$$

and this should be analytic in the strip $-k_{2}<\operatorname{Im} w<k_{2} \cos \alpha$. $x$ to read

We can treat equation (2.6) in much the same fashion. We extend it for all

$$
F_{2}(x)=\phi_{2}(x)-\frac{f_{1}(x)}{2}+\frac{\mathrm{i}}{4}\left(\frac{\partial^{2}}{\partial x^{2}}+k^{2}\right) \int_{0}^{\infty} H_{0}^{(1)}\left[k\left|x-x^{\prime}\right|\right] f_{2}\left(x^{\prime}\right) \mathrm{d} x^{\prime}
$$

where $\phi_{2}(x) \equiv 0, \quad x<0$

$$
\equiv \mathrm{i} k_{y} \exp \left(\mathrm{i} x k_{x}\right), \quad x>0
$$

$$
F_{2}(x) \equiv 0, \quad x>0
$$

and $f_{1}(x)=f_{2}(x) \equiv 0, \quad x<0$.

Now due to the assumption which we made about the behavior of the integral in the neighborhood of the origin, we may calculate the Fourier transform of equation (3.2), integrating by parts to eliminate the second $x$-derivative and noting that there are no contributions from $\pm \infty$. Here we get

$$
\hat{F}_{2}(w)=\frac{k_{y}}{w-k_{y}}-\frac{f_{1}(w)}{2}+\frac{i \sqrt{k^{2}-w^{2}} \hat{f}_{2}(w)}{2}
$$

which is again analytic in the strip $-k_{2}<\operatorname{Im} w<k_{2} \cos \alpha$. It is from (3.1) and (3.3) that we will show that it is possible to determine acceptable $f_{1}(x)$ and $f_{2}(x)$ for $x>0$ as well as $F_{1}(x)$ and $F_{2}(x)$ for $x<0$.

\section{The Wiener-Hopf Separation}

We shall now show that equations (3.1) and (3.3) can be separated into components which are analytic in the lower half-plane $\operatorname{Im} w<k_{2} \cos \alpha$ and the upper half-plane $\operatorname{Im} w>-k_{2}$ at the expense of producing a second pair of integral equations of the Cauchy type along a branch cut drawn from $-k$. It will turn out, due to the functions which are now encountered, that this second pair can be solved explicitly by a method due to Carleman [2] and we will thus be able 
to find the Fourier transforms of $f_{1}(x)$ and $f_{2}(x)$. They are not of the simple structure of those encountered in the original Sommerfeld problems.

In this section we derive the separation of equation (3.1). We first rewrite this equation as

$$
\sqrt{k+w} F_{1}(w)=\frac{\sqrt{k+w}}{i\left(w-k_{x}\right)}+\frac{i}{2} \frac{\hat{f}_{1}(w)}{\sqrt{k-w}}-\frac{\sqrt{k+w} f_{2}(w)}{2} .
$$

The second term may be directly decomposed into two terms, one of which displays the branch point singularity and is therefore analytic in the upper half plane $\operatorname{Im} w>-k_{2}$. The other shows the dependence on the pole $w=k_{x}$ and is therefore analytic in the lower half-plane $\operatorname{Im} w<k_{2} \cos \alpha$. That is, we have

$$
\begin{aligned}
\sqrt{k+w} \hat{f}_{1}(w)-\frac{\sqrt{k+w}-\sqrt{k+k_{x}}}{\mathrm{i}\left(w-k_{x}\right)}= & \frac{\sqrt{k+k_{x}}}{\mathrm{i}\left(w-k_{x}\right)}+\frac{\mathrm{i}}{2} \frac{f_{1}(w)}{\sqrt{k-w}} \\
& -\frac{f_{2}(w) \sqrt{k+w}}{2} .
\end{aligned}
$$

The left side of this equation is now analytic in the upper half-plane $\operatorname{Im} w>-k_{2}$ and the first two terms of the right side are analytic in the lower half-plane Im $w$ $<k_{2} \cos \alpha$ and these three terms are analytic in a common strip. The last term is only analytic in this strip and the Wiener-Hopf separation process is still not complete.

To separate $f_{2}(w) \sqrt{k+w}$ into two parts, each of which is regular in the appropriate half-plane, we need the Cauchy integral theorem. First we make a unique determination of the $\sqrt{k+w}$ by drawing a branch cut from $-k$ to $-\infty$ $\exp [i \arg k$ ], $0<\arg k<\pi / 2$, in the lower half plane (see Fig. 1).

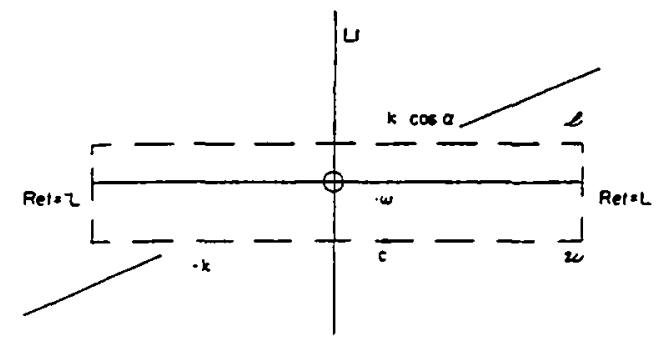

Fig. 1

This branch cut is an extension of the line segment drawn from $-k$ to $k$ in the lower half-plane. Along the lower edge of the cut (as a result of our original choice of $\sqrt{k^{2}-w^{2}}$ ) we have $\sqrt{k+w}=e^{-i \pi / 2} \sqrt{-k-w}$ while on the upper edge we have $\mathrm{e}^{i \pi / 2} \sqrt{-k-w}$. Now in the strip $-k_{2}<\operatorname{Im} w<k_{2} \cos \alpha$ (see Fig. 1) we apply the Cauchy integral theorem to $\sqrt{k+w} f_{2}(w)$ to obtain

$$
\sqrt{k+w} f_{2}(w)=\frac{1}{2 \pi i} \int_{c} \frac{\sqrt{k+t} f_{2}(t) \mathrm{d} t}{t-w} .
$$


Since we have assumed, subject to verification, that $f_{2}(t)=O\left(t^{-S / 4}\right),|t| \rightarrow \infty$ in the appropriate lower half-plane, the integrals along $t=L+\mathrm{i} \sigma$ and $t=-L+$ $\mathrm{i} \sigma,-k_{2}<\sigma<k_{2} \cos \alpha$ vanish as $L \rightarrow \infty$. This gives us for $w$ in the strip of analyticity

$$
\sqrt{k+w} f_{2}(w)=\frac{1}{2 \pi \mathrm{i}} \int_{t} \frac{\sqrt{k+t}}{t-w} \hat{f}_{2}(t) \mathrm{d} t+\frac{1}{2 \pi \mathrm{i}} \int_{u} \frac{\sqrt{k+t}}{t-w} \hat{f}_{2}(t) \mathrm{d} t
$$

where

$$
\int_{l} \frac{\sqrt{k+t} \hat{f}_{2}(t)}{t-w} \mathrm{~d} t=\int_{\infty+\mathrm{i}\left(k_{2} \cos \alpha-\epsilon\right)}^{-\infty+\mathrm{i}\left(k_{2} \cos \alpha-\ell\right)} \frac{\sqrt{k+t} \hat{f}_{2}(t)}{t-w} \mathrm{~d} t
$$

and

$$
\int_{u} \frac{\sqrt{k+t} f_{2}(t)}{t-w} \mathrm{~d} t=\int_{-\infty-\mathrm{i}\left(k_{2}-\varepsilon\right)}^{\infty-\mathrm{i}\left(k_{2}-\varepsilon\right)} \frac{\sqrt{k+t}}{t-w} f_{2}(t) \mathrm{d} t
$$

where $\varepsilon<k_{2}(1+\cos \alpha) / 2, \varepsilon$ has been chosen to guarantee that the path $l$ is below the pole $k \cos \alpha$, the path $u$ is above the branch point $-k$ and the path $l$ is above the path $u$. Clearly, with the behavior we have assumed for $\hat{f}_{2}(t),(4.2)$ is analytic in the lower half plane $\operatorname{Im} w<k_{2} \cos \alpha$ and (4.3) is analytic in the upper half plane $\operatorname{Im} w>-k_{2}$. Hence the final separation of equation (3.1) is now

$$
\begin{aligned}
& \sqrt{k+w} \hat{F}_{1}(w)-\frac{\sqrt{k+w}-\sqrt{k+k_{x}}}{\mathrm{i}\left(w-k_{x}\right)}+\frac{1}{4 \pi \mathrm{i}} \int_{u} \frac{\sqrt{k+t} f_{2}(t) \mathrm{d} t}{t-w} \\
& =\frac{\sqrt{k+k_{x}}}{\mathrm{i}\left(w-k_{x}\right)}+\frac{\mathrm{i}}{2} \frac{\hat{f}_{1}(w)}{\sqrt{k-w}}-\frac{1}{4 \pi \mathrm{i}} \int_{t} \frac{\sqrt{k+t} f_{2}(t) \mathrm{d} t}{t-w}
\end{aligned}
$$

where now the left side of (4.4) is analytic in the upper half plane $\operatorname{Im} w>-k_{2}$ and the right side is analytic in the lower half plane $\operatorname{Im} w<k_{2} \cos \alpha$. The argument of Wiener and Hopf now tells us that each side of (4.4) is an entire function of $w$, which we shall label $E_{1}(w)$.

In a similar fashion we obtain, upon separating equation (3.3)

$$
\begin{aligned}
& \frac{\hat{F}_{2}(w)}{\sqrt{k+w}}-\frac{k_{y}}{\left(w-k_{x}\right)}\left[\frac{1}{\sqrt{k+w}}-\frac{1}{\sqrt{k+k_{x}}}\right]+\frac{1}{4 \pi \mathrm{i}} \int \frac{f_{1}(t) \mathrm{d} t}{\sqrt{k+t}(t-w)} \\
& =\frac{k_{y}}{\left(w-k_{x}\right) \sqrt{k+k_{x}}}-\frac{1}{4 \pi \mathrm{i}} \int \frac{f_{1}(t) \mathrm{d} t}{\sqrt{k+t}(t-w)}+\frac{\mathrm{i} \sqrt{k-w}}{2} f_{2}(w) \\
& =E_{2}(w)
\end{aligned}
$$

where the left and right sides of (4.5) have the same analyticity properties as equation (4.4), $E_{2}(w)$ is an entire function which we shall evaluate with $E_{1}(w)$ in the next section. 


\section{The Preliminary Determination of $E_{1}(w)$ and $E_{2}(w)$}

In the scalar Wiener-Hopf problems, the determination of the entire function of separation is affected by observing the Riemann-Lebesgue lemma for the unknown Fourier transforms and the asymptotic behavior of the analytic functions that were encountered. Now we have an added ingredient, namely the integrals along $l$ and $u$.

We start by examining the integrals in (4.4). For example

$$
\int_{t} \frac{\sqrt{k+t}}{t-w} f_{2}(t) \mathrm{d} t=\int_{\infty}^{-\infty} \frac{\sqrt{k+\tau+\mathrm{i}\left(k_{2} \cos \alpha-\varepsilon\right)} \hat{f}_{2}\left[\tau+\mathrm{i}\left(k_{2} \cos \alpha-\varepsilon\right)\right] \mathrm{d} \tau}{\tau+\mathrm{i}\left(k_{2} \cos \alpha-\varepsilon\right)-w} .
$$

Hence we can employ the Schwarz inequality since

$$
\sqrt{\tau+k+\mathrm{i}\left(k_{2} \cos \alpha-\varepsilon\right)} \hat{f}_{2}\left[\tau+\mathrm{i}\left(k_{2} \cos \alpha-\varepsilon\right)\right]=O\left(\tau^{-3 / 4}\right), \quad|\tau| \rightarrow \infty
$$

and we obtain

$$
\begin{aligned}
& \left|\int_{t} \frac{\sqrt{k+t} \hat{f}_{2}(t) \mathrm{d} t}{t-w}\right|^{2} \\
& \leqslant \int_{-\infty}^{\infty}\left|\sqrt{k+\tau+\mathrm{i}\left(k_{2} \cos \alpha-\varepsilon\right)} f\left[\tau+\mathrm{i}\left(k_{2} \cos \alpha-\varepsilon\right)\right]\right|^{2} \mathrm{~d} \tau \\
& \quad \times \int_{-\infty}^{\infty} \frac{\mathrm{d} \tau}{\left|\tau+\mathrm{i}\left(k_{2} \cos \alpha-\varepsilon\right)-w\right|^{2}} . \\
& =A /\left|k_{2} \cos \alpha-\varepsilon-v\right|, \quad w=u+\mathrm{i} v
\end{aligned}
$$

where $A$ is a constant. Clearly then the integral along $l$ is analytic and bounded for $\operatorname{Im} w<k_{2} \cos \alpha-\varepsilon$. However, the right side of the inequality is independent of $u$ and does not vanish when $u^{2}+v^{2} \rightarrow \infty$ in the full neighborhood of infinity when $v<k_{2} \cos \alpha-\varepsilon$. In a similar fashion we can show that the integral along $u$ is also analytic and bounded in the upper half plane $\operatorname{Im} w>-\left(k_{2}-\varepsilon\right)$. The first two terms on the left side of (4.4) vanish when $|w| \rightarrow \infty, \operatorname{Im} w>-\left(k_{2}-\varepsilon\right)$ and the third term is bounded in the same upper half-plane. We also have that the first two terms on the right side vanish when $|w| \rightarrow \infty, \operatorname{Im} w<k_{2} \cos \alpha-\varepsilon$ and the third term is bounded. Hence $E_{1}(w)$ is bounded everywhere and therefore by Liouville's theorem, $E_{1}(w)$ is a constant which we label $E_{1}$. In a similar fashion we find that $E_{2}(w)$ is a constant $E_{2}$. This determination of $E_{1}(w)$ and $E_{2}(w)$ now gives us two equations for $f_{1}(w)$ and $\vec{f}_{2}(w)$. It remains for us to cast equations (4.4) (4.5) into a usable form as well as to evaluate $E_{1}$ and $E_{2}$. It is unfortunate that the estimates are not sufficiently strong to give a more determinate value for $E_{1}$ and $E_{2}$.

\section{Simplification of Equations (4.4) and (4.5)}

If we could deform the path $l$ into an integral taken along a real variable, this would be of much assistance in our future development. This cannot be done 
directly in the present case, since $f_{2}(w)$ is not analytic in the upper half-plane. In order to circumvent this difficulty and simultaneously produce a useable form, we rewrite (4.1) as

$$
f_{2}(w) \sqrt{k+w}-\frac{1}{2 \pi i} \int_{u} \frac{\sqrt{k+t} \hat{f}_{2}(t) \mathrm{d} t}{t-w}=\frac{1}{2 \pi i} \int_{t} \frac{\sqrt{k+t} \hat{f}_{2}(t) \mathrm{d} t}{t-w} .
$$

Now since $f_{2}(t)$ is analytic below $u$, we shall examine the integral

$$
\int_{\Gamma(R)} \frac{\sqrt{k+t}}{t-w} f_{2}(t) \mathrm{d} t
$$

where $\Gamma(R)$ is the path which encloses the branch cut drawn from $-k$ and is connected to $u$ by circular arcs of radius $R$ (see Fig. 2). Let us note that the

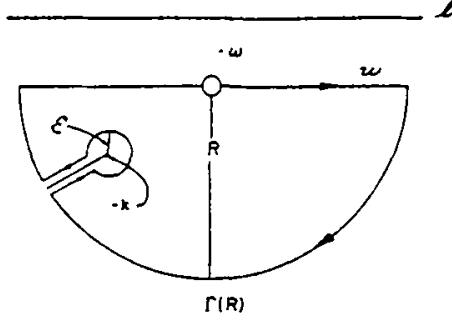

Fig. 2

integral along th circle of radius $\varepsilon$ drawn about the branch point $-k$ does not make a contribution when $\varepsilon \rightarrow 0$ since $f_{2}(t)$ is analytic in the neighborhood of $t=$ $-k$ and $(t+k)^{1 / 2}$ is $O\left(\varepsilon^{1 / 2}\right)$ in this neighborhood. Furthermore, the integral along the circular arcs of radius $R$ does not make any contribution when $R \rightarrow \infty$ because we have assumed that $\hat{f}_{2}(t)=O\left(|t|^{-5 / 4}\right),|t| \rightarrow \infty, \operatorname{Im} t<k_{2} \cos \alpha$. This then leaves us with the integral along $u$ and integrals along both sides of the branch cut which, of course, add to zero in virtue of the Cauchy integral theorem. Hence the integral along $u$ is the negative of the integral along the branch cut taken in the clockwise sense.

Now because of our choice of the branch of $\sqrt{k+t}$, we have $\sqrt{k+t}=$ $-\mathrm{i} \sqrt{-k-t}$ on the lower side of the cut and $\sqrt{k+t}=\mathrm{i} \sqrt{-k-t}$ on the upper side of the cut where now we have arg $\sqrt{-k-t}=\beta$ when $t$ is on the cut. Along the cut we have then, when $R \rightarrow \infty$

$$
\int_{u} \frac{\sqrt{k+t} \hat{f}_{2}(t) \mathrm{d} t}{t-w}=-2 \mathrm{i} \int_{-k}^{-\infty \exp \mathrm{i} \beta} \frac{\sqrt{-k-t} \hat{f}_{2}(t) \mathrm{d} t}{t-w} .
$$

With the substitution $t=-\tau \exp i \beta$, this last integral may be rewritten as

$$
-2 \mathrm{i} \int_{|k|}^{\infty} \frac{\sqrt{-k+\tau \operatorname{expi} \beta} f_{2}(-\tau \operatorname{expi} \beta) \operatorname{expi} \beta \mathrm{d} \tau}{\tau \operatorname{expi} \beta+w}
$$

where now the integration is taken along the real variable $\tau$. Hence the right side of $(4.4)$ becomes 


$$
\begin{aligned}
& \frac{\sqrt{k+k_{x}}}{\mathrm{i}\left(w-k_{x}\right)}+\frac{\mathrm{i} \hat{f}_{1}(w)}{2 \sqrt{k-w}}-\frac{1}{2} f_{2}(w) \sqrt{k+w} \\
& -\frac{1}{2 \pi} \int_{|k|}^{\infty} \frac{\sqrt{-k+\tau \operatorname{expi} \beta} f_{2}(-\tau \operatorname{expi} \beta) \mathrm{d} \tau}{\tau+w(\exp -\mathrm{i} \beta)}=E_{1}
\end{aligned}
$$

valid in the lower half-plane $\operatorname{Im}<k_{2} \cos \alpha, w$ not on the cut from $-k$.

In a similar fashion, when we observe that $\hat{f}_{1}(t)=O\left(|t|^{-1 / 4}\right),|t| \rightarrow \infty$, $\operatorname{Im} w<k_{2} \cos \alpha$, we can rewrite (4.5) as

$$
\begin{aligned}
& \frac{k_{y}}{\left(w-k_{x}\right) \sqrt{k+k_{x}}}+\frac{\mathrm{i} \sqrt{k-w}}{2} \hat{f}_{2}(w)-\frac{f_{1}(w)}{2 \sqrt{k+w}} \\
& +\frac{1}{2 \pi} \int_{|k|}^{\infty} \frac{f_{1}(-\tau \exp \mathrm{i} \beta) \mathrm{d} \tau}{\sqrt{-k+\tau \exp \mathrm{i} \beta}[\tau+w(\exp -\mathrm{i} \beta)]}=E_{2}
\end{aligned}
$$

with the same restriction as for equation (6.1).

VII The Reduction of Equations (6.1) and (6.2) to a System of Carleman Type Integral Equations

Equations (6.1) and (6.2) were derived subject to the fact that $w$ was not on the branch cut from $-k$. We now examine the combination of terms

$$
\frac{-\hat{f}_{2}(w) \sqrt{k+w}}{2}-\frac{1}{2 \pi} \int_{|k|}^{\infty} \frac{\sqrt{-k+\tau \mathrm{e}^{\mathrm{i} \beta}} \hat{f}_{2}\left(-\tau \mathrm{e}^{\mathrm{i} \beta}\right) \mathrm{d} \tau}{\tau+w \mathrm{e}^{-\mathrm{i} \beta}}
$$

when $w$ is on the cut. The situation we face becomes more familiar if we replace $w$ by $-w$. Hence we consider

$$
\frac{-f_{2}(-w) \sqrt{k-w}}{2}-\frac{1}{2 \pi} \int_{|k|}^{\infty} \frac{\sqrt{-k+\tau \mathrm{e}^{\mathrm{i} \beta}} f_{2}\left(-\tau \mathrm{e}^{\mathrm{i} \beta}\right) \mathrm{d} \tau}{\tau-w \mathrm{e}^{-\mathrm{i} \beta}}
$$

With $\sqrt{k-w}=\sqrt{k}=|k| \mathrm{e}^{(\mathrm{i} \beta) / 2}$ when $w=0$, we note that $\sqrt{k-w}=-\mathrm{i} \sqrt{w-k}$ when $w$ is on the top side of the branch cut and is $i \sqrt{w-k}$ when $w$ is on the bottom side of the cut. On the other hand, if $w=u+i v$, the integral in (7.1) may be written as

$$
\begin{aligned}
& -\frac{1}{2 \pi} \int_{|k|}^{\infty} \\
& \frac{\sqrt{-k+\tau \mathrm{e}^{\mathrm{i} \beta}} f_{2}\left(-\tau \mathrm{e}^{\mathrm{i} \beta}\right)[\tau-(u \cos \beta+v \sin \beta)+\mathrm{i}(v \cos \beta-u \sin \beta] \mathrm{d} t}{\tau^{2}-2 \tau(u \cos \beta+v \sin \beta)+\left(u^{2}+v^{2}\right)} .
\end{aligned}
$$

Now a point on the branch cut from $k$ is on the straight line $v \cos \beta-u \sin \beta=0$, $u>|k| \cos \beta, v>|k| \sin \beta$. If $v \cos \beta-u \sin \beta<0, u$ approaches a point on the bottom of the branch cut. In this case (7.2) has the limit 


$$
\begin{aligned}
& -\frac{1}{2 \pi} \oint_{|k|}^{\infty} \frac{\sqrt{-k+\tau \mathrm{e}^{\mathrm{i} \beta}} \hat{f}_{2}\left(-\tau \mathrm{e}^{\mathrm{i} \beta} \mathrm{d} \tau\right.}{\tau-(u \cos \beta+v \sin \beta)} \\
& +\frac{\mathrm{i} \pi}{2 \pi} \sqrt{-k+\mathrm{e}^{\mathrm{i} \beta}(u \cos \beta+v \sin \beta)} f_{2}\left[-(u \cos \beta+v \sin \beta) \mathrm{e}^{\mathrm{i} \beta}\right]
\end{aligned}
$$

where now $v \cos \beta-u \sin \beta=0$ when $u$ and $v$ are coordinates on the cut from $k$. (7.3), in turn, simplifies to

$$
-\frac{1}{2 \pi} \oint_{|k|}^{\infty} \frac{\sqrt{-k+\tau \mathrm{e}^{\mathrm{i} \beta}} f_{2}\left(-\tau \mathrm{e}^{\mathrm{i} \beta}\right) \mathrm{d} \tau}{\tau-|w|}+\frac{\mathrm{i}}{2} \sqrt{-k+|w| \mathrm{e}^{\mathrm{i} \beta}} f_{2}\left[-\mathrm{e}^{\mathrm{i} \beta}|w|\right] .
$$

This last form follows from an application of the Poisson integral formula for the half plane. The integral is taken in the sense of a Cauchy principal value. With the result of (7.3) we note that the limit of (7.1) when $w$ approaches the cut from $k$ on the bottom we have

$$
-\frac{1}{2 \pi} \oint_{|k|}^{\infty} \frac{\sqrt{-k+\tau \mathrm{e}^{\mathrm{i} \beta}} \hat{f}_{2}\left(-\tau \mathrm{e}^{\mathrm{i} \beta}\right) \mathrm{d} \tau}{\tau-|w|}=-\frac{\mathrm{e}^{(\mathrm{i} \beta) / 2}}{2 \pi} \oint_{|k|}^{\infty} \frac{\sqrt{\tau-|k|} \hat{f}_{2}\left(-\tau \mathrm{e}^{\mathrm{i} \beta}\right) \mathrm{d} \tau}{\tau-|w|} .
$$

Finally we note that if $w$ approaches the top side of the cut from $k$ we obtain (7.4) once again since the sign of the term external to the integral in (7.1) is now reversed and of course so is $v \cos \beta-u \sin \beta$ when this limit is taken.

Hence on the cut from $k$ (either side)

$$
\frac{-\sqrt{k+k_{x}}}{\mathrm{i}\left(w+k_{x}\right)}+\frac{\mathrm{i} \hat{f}_{1}(-w)}{2 \sqrt{k+w}}-\frac{\mathrm{e}^{(\mathrm{i} \beta) / 2}}{2 \pi} \oint_{|k|}^{\infty} \frac{\left.\sqrt{\tau-|k|} \hat{f}_{2}(-\tau) \operatorname{expi} \beta\right) \mathrm{d} \tau}{\tau-|w|}=E_{1} .
$$

Upon putting $w=|w|(\operatorname{expi} \beta)$ and $k=|k|(\operatorname{expi} \beta)$ we have

$$
\begin{aligned}
& \frac{(\exp \mathrm{i} \beta)}{2 \pi} \oint_{|k|}^{\infty} \frac{\sqrt{\tau-|k|} \hat{f}_{2}(-\tau \exp \mathrm{i} \beta) \mathrm{d} \tau}{\tau-|w|} \\
& =-E_{1}+\frac{\mathrm{i} \sqrt{|k|(1+\cos \alpha)}}{|w|+|k| \cos \alpha}+\frac{\mathrm{i}}{2} \frac{f_{1}(-|w| \exp \mathrm{i} \beta)}{\sqrt{|k|+|w|}}
\end{aligned}
$$

for $|w|>|k|$.

There is a second such integral equation of the general form (7.6) which we derive from (4.5) with the same methods which we just employed. Here we have

$$
\begin{aligned}
& (\operatorname{expi} \beta) \hat{f}_{2}(-|w| \exp \mathrm{i} \beta) \sqrt{|w|+|k|} \\
& =\frac{\mathrm{i}}{\pi} \oint_{|k|}^{\infty} \frac{\hat{f}_{1}(-\tau \exp \mathrm{i} \beta) \mathrm{d} \tau}{\sqrt{\tau-|k|}(\tau-|w|)}-\frac{2 \mathrm{i} \sqrt{|k|} \sin \alpha}{(|w|+|k| \cos \alpha) \sqrt{1+\cos \alpha}}+E_{2} .
\end{aligned}
$$


VIII The Reduction of Equations (7.6) and (7.7) to a Single Integral Equation of the Cauchy Type and its Solution

It may be tempting to substitute either equation (7.6) into (7.7) and obtain an integral equation for $\vec{f}_{2}(-\tau \exp i \beta)$ or perhaps do the reverse and substitute (7.7) into (7.6) and obtain an integral equation for $\hat{f}_{1}(-\tau \operatorname{exp~i} \beta)$. Unfortunately, in either case we cannot use the Bertrand-Poincare formula to effect any simplification since the Fourier transforms $\hat{f}_{1}$ and $\hat{f}_{2}$ do not meet the conditions needed to apply it. Hence we proceed more directly. We first show that we may invert the integral operator in equation (7.6) and thereby find $f_{2}(-\tau \operatorname{exp~i} \beta)$ explicitly. We may therefore combine the inverted equation (7.6) with (7.7) to produce an integral equation for $\hat{f}_{1}(-\tau \exp \mathrm{i} \beta)$.

We will first show that we can solve equation (7.6) explicitly for $\hat{f}_{2}$ ( $-Q \operatorname{expi} \beta$ ). This will provide the necessary reduction to enable us to obtain an integral equation for $\hat{f}_{1}$ ( $\left.-\varrho \operatorname{expi} \beta\right)$ which, in turn, can be solved explicitly. The same method can be used to invert the equation (7.6) and solve the integral equation for $f_{1}(-\varrho \operatorname{expi} \beta)$. We start with the observation that the integral operator on the left side of equation (7.6) may be inverted to give $[2,4]$

$$
\begin{aligned}
& (\operatorname{expi} \beta) \hat{f}_{2}(-|w| \operatorname{expi} \beta) \\
& =-\frac{1}{\pi} \oint_{|k|}^{\infty}\left[-2 E_{1}+2 \mathrm{i} \frac{\sqrt{|k|(1+\cos \alpha)}}{\tau+|k| \cos \alpha}+\frac{\mathrm{i} \hat{f}_{1}(-\tau \exp \mathrm{i} \beta)}{\sqrt{\tau+|k|}}\right] \\
& \cdot \frac{\mathrm{d} \tau}{\sqrt{\tau-|k|}(\tau-|w|)}+\frac{C_{1}}{|w|-|k|} .
\end{aligned}
$$

The term $C_{1} /(|w|-|k|)$ is a solution of the homogeneous operator

$$
\oint_{|k|}^{\infty} \frac{\sqrt{\tau-|k|} \hat{f}_{2}(-\tau \operatorname{expi} \beta) \mathrm{d} \tau}{\tau-|w|}=0, \quad|w|>|k| .
$$

However, such a solution would imply that $\hat{f}_{2}[-w]$ would have a pole in the upper half-plane and therefore $C_{1}=0$. We note further that the integral involving $E_{1}$ vanishes. Hence equation (8.1) reduces to

$$
\begin{aligned}
& (\exp \mathrm{i} \beta) \hat{f}_{2}(-|w| \exp \mathrm{i} \beta) \\
& =\frac{-\mathrm{i}}{\pi} \oint_{|k|}^{\infty} \frac{\hat{f}_{1}(-\tau \exp \mathrm{i} \beta) \mathrm{d} \tau}{\sqrt{\tau^{2}-|k|^{2}}(\tau-|w|)}+\frac{2 \mathrm{i}}{|w|+|k| \cos \alpha}
\end{aligned}
$$

upon evaluating the second integral in (8.1).

From equation (7.7) we also have an explicit expression for $(\operatorname{expi} \beta) \hat{f}_{2}(-\tau \operatorname{expi} \beta)$ and it is now possible to eliminate this quantity to obtain

$$
\begin{aligned}
& \frac{\mathrm{i}}{\pi} \oint_{|k|}^{\infty} \frac{f_{1}(-\varrho \exp \mathrm{i} \beta) \mathrm{d} \varrho}{\sqrt{\varrho-|k|}(\varrho-\tau)}\left[\frac{1}{\sqrt{\varrho+|k|}}+\frac{1}{\sqrt{\tau+|k|}}\right] \\
& =\frac{2 \mathrm{i}}{\tau+|k| \cos \alpha}\left[1+\frac{\sqrt{|k|} \sin \alpha}{\sqrt{\tau+|k|} \sqrt{1+\cos \alpha}}\right]+\frac{E_{2}}{\sqrt{\tau+|k|}} .
\end{aligned}
$$


With the substitutions $\varrho=|k|\left(2 \lambda^{2}-1\right)$ and $\tau=|k|\left(2 \sigma^{2}-1\right)$, equation (8.3) becomes

(8.3a) $\frac{1}{\pi} \oint_{1}^{\infty} \frac{\hat{f}_{1}\left[-|k|(\exp \mathrm{i} \beta)\left(2 \lambda^{2}-1\right)\right] \mathrm{d} \lambda}{\sqrt{\lambda^{2}-1}(\lambda-\sigma)}=\frac{1}{\sigma-\sin \frac{\alpha}{2}}-\mathrm{i} E_{2} \sqrt{\frac{k}{2}}$.

We have already seen that such an equation has a solution of the form

(8.4) $\quad f_{1}\left[-|k|(\exp i \beta)\left(2 \lambda^{2}-1\right)\right]$

$$
=\frac{-\sqrt{\lambda+1}}{\pi} \oint_{1}^{\infty}\left[\frac{2}{\sigma-\sin \frac{\alpha}{2}}-\mathrm{i} E_{2} \sqrt{\frac{k}{2}}\right] \frac{\sqrt{\sigma-1}}{(\sigma-\lambda)} \mathrm{d} \sigma .
$$

But clearly the part of the solution containing the factor $E_{2}$ diverges and therefore in order to invoke the regularity condition on $\hat{f}_{1}\left[-|k|(\operatorname{exp~i} \beta)\left(2 \lambda^{2}-1\right)\right]$, we must take $E_{2}=0$. The integral in (8.4) therefore reduces to

$$
\begin{aligned}
& \hat{f}_{1}\left[-|k|(\operatorname{expi} \beta)\left(2 \lambda^{2}-1\right)\right]=\frac{-2 \sqrt{\lambda+1}}{\pi} \oint_{1}^{\infty} \frac{\sqrt{\sigma-1} \mathrm{~d} \sigma}{\left(\sigma-\sin \frac{\alpha}{2}\right)(\sigma-\lambda)} \\
& =\frac{-2 \sqrt{\lambda+1}}{\pi\left(\lambda-\sin \frac{\alpha}{2}\right)} \oint^{\infty}\left[\frac{1-\sin \frac{\alpha}{2}}{\sigma-\sin \frac{\sigma}{2}}-\frac{1-\lambda}{\sigma-1}\right] \frac{\mathrm{d} \sigma}{\sqrt{\sigma-1}} .
\end{aligned}
$$

But the second integral vanishes since $\lambda>1$. The substitution $\sigma-1=Q^{2}$ reduces the first integral to

$$
\hat{f}_{1}\left[-|k|(\operatorname{expi} \beta)\left(2 \lambda^{2}-1\right)\right]=-\frac{\sqrt{\lambda+1} \sqrt{1-\sin \frac{\alpha}{2}}}{\lambda-\sin \frac{\alpha}{2}} .
$$

The determination of $f_{2}[-\tau \operatorname{expi} \beta]$ is now elementary. We turn to equation (7.7) and find that when $|w|=|k|\left(2 \lambda^{2}-1\right)$ and $\tau=|k|\left(2 \sigma^{2}-1\right)$ we have

$$
\begin{aligned}
& \sqrt{2|k|} \lambda(\operatorname{expi} \beta) f_{2}\left[-|k|(\operatorname{expi} \beta)\left(2 \lambda^{2}-1\right)\right] \\
& =-\frac{\mathrm{i}}{\pi} \sqrt{\frac{2}{|k|}} \oint_{i}^{\infty} \frac{\sqrt{1-\sin \frac{\alpha}{2}} \sigma \mathrm{d} \sigma}{\left(\sigma-\sin \frac{\alpha}{2}\right)\left(\sigma^{2}-\lambda^{2}\right) \sqrt{\sigma-1}}-\mathrm{i} \sqrt{\frac{2}{|k|}} \frac{\sin \frac{\alpha}{2}}{\lambda^{2}-\sin ^{2} \frac{\alpha}{2}}
\end{aligned}
$$


We decompose the integral into three terms, that is

$$
\begin{aligned}
& \oint_{1}^{\infty} \frac{\sigma \mathrm{d} \sigma}{\sqrt{\sigma-1}\left(\sigma-\sin \frac{\alpha}{2}\right)\left(\sigma^{2}-\lambda^{2}\right)} \\
& =\oint_{i}^{\infty} \frac{\mathrm{d} \sigma}{\sqrt{\sigma-1}}\left[\frac{A}{\sigma-\sin \frac{\alpha}{2}}+\frac{B}{\sigma-\lambda}+\frac{C}{\sigma+\lambda}\right]
\end{aligned}
$$

where $A=\frac{\sin \frac{\alpha}{2}}{\sin ^{2} \frac{\alpha}{2}-\lambda^{2}}, \quad B=\frac{1}{2\left(\lambda-\sin \frac{\alpha}{2}\right)}$

and $\quad C=-\frac{1}{2\left(\lambda+\sin \frac{\alpha}{2}\right)}$

(8.6) in turn reduces to

$$
\frac{\pi A}{\sqrt{1-\sin \frac{\alpha}{2}}}+\frac{\pi C}{\sqrt{1+\lambda}}
$$

and hence, (8.5) simplifies to

(8.7) $\quad(\operatorname{expi} \beta) \hat{f}_{2}\left[-|k|(\operatorname{expi} \beta)\left(2 \lambda^{2}-1\right)\right]$

$$
=\frac{i \sqrt{1-\sin \frac{\alpha}{2}}}{2 \lambda|k| \sqrt{1+\lambda}\left(\lambda+\sin \frac{\alpha}{2}\right)} .
$$

IX Some Properties of $\phi(x, 0+)$ and $\frac{8 \phi}{\theta x}(x, 0-)$

We are now in a position to discuss some of the properties of $f_{1}(x)$ and $f_{2}(x)$. We basically have their Fourier transforms in (8.9) and (8.10). If we make the substitution $w=k\left(2 \lambda^{2}-1\right)$, we find that

(9.1) $f_{1}(w)=-\frac{\sqrt{1-\sin \alpha / 2} \sqrt{1+\sqrt{(k-w) / 2 k}}}{\sqrt{(k-w) / 2 k}-\sin \alpha / 2}$. 
(9.1) is analytic in the lower half plane $\operatorname{Im} w<\operatorname{Im} k \cos \alpha$ and is $O\left(w^{-1 / 4}\right)$ when $|w| \rightarrow \infty$ in this half-plane. We therefore know that $f_{1}(x)=O\left(x^{-3 / 4}\right), x \rightarrow 0$ which verifies one of our assumptions. If we examine $(8.10)$ with the same substitution we find that

$$
f_{2}(w)=\frac{i \sqrt{1-\sin \alpha / 2}}{2 k \sqrt{(k-w) / 2 k} \sqrt{1+\sqrt{(k-w) / 2 k}}[\sqrt{(k-w) / 2 k}+\sin \alpha / 2]}
$$

We note now that $\hat{f}_{2}(w)$ is analytic in the lower half-plane $\operatorname{Im} w<\operatorname{Im} k$ and is $O\left(w^{-5 / 4}\right)$ when $|w| \rightarrow \infty$ in this half-plane. This then verifies that $f_{2}(x)=$ $O\left(x^{1 / 4}\right), x \rightarrow 0+.(9.2)$ may be written in an alternate form as

$$
f_{2}(w)=\frac{i \sqrt{1-\sin \alpha / 2} \sqrt{1-\sqrt{(k-w) / 2 k}}}{\sqrt{k^{2}-w^{2}}[\sqrt{(k-w) / 2 k}+\sin \alpha / 2]}
$$

Finally, we shall examine $\phi(x, 0)$ for $x<0$ and $x>0, y= \pm 0$. To this end, we rewrite equation (2.1) as a Fourier integral representation, that is

$$
\begin{aligned}
\phi(x, \pm 0)=\exp \left(\mathrm{i} x k_{x}\right) & +\frac{\mathrm{i}}{4 \pi} \int_{\mathscr{S}} \frac{f_{1}(w) \exp (\mathrm{i} w x)}{\sqrt{k^{2}-w^{2}}} \mathrm{~d} w \\
& \pm \frac{1}{4 \pi} \int_{\mathscr{S}} \hat{f}_{2}(w) \exp (\mathrm{i} w x) \mathrm{d} w
\end{aligned}
$$

where $\mathscr{L}$ is a path in the common strip of analyticity of $f_{1}(w)$ and $f_{2}(w)$, that is, in the strip $-\operatorname{Im} k<\operatorname{Im} w<\operatorname{Im} k \cos \alpha$.

We can dispense with the determination of $\phi(x, 0), x<0$ very easily. We close $\mathscr{L}$ in the lower half-plane taking into account the branch cut drawn from $-k$. Since $f_{1}$ and $f_{2}$ are regular in the lower half-plane, the integral containing $\hat{f}_{2}(w)$ vanishes and we are left with

$$
\begin{aligned}
\phi(x, 0)= & \exp \left(\mathrm{i} x k_{x}\right) \\
& +\frac{1}{2 \pi} \int_{i}^{\infty} \frac{\sqrt{1-\sin \alpha / 2} \sqrt{1+\sqrt{(1+\tau) / 2}} \exp (-\mathrm{i} x k \tau) \mathrm{d} \tau}{\sqrt{\tau^{2}-1}[\sqrt{(1+\tau) / 2}-\sin \alpha / 2]}
\end{aligned}
$$

upon using the same methods which were used in Section VII. The substitution $\tau=\cosh \psi$ will remove the radical signs to give us

$$
\begin{aligned}
\phi(x, 0)= & \exp \left(\mathrm{i} x k_{x}\right) \\
& +\frac{\sqrt{1-\sin \alpha / 2}}{\sqrt{2} \pi} \int_{0}^{\infty} \frac{\exp (-\mathrm{i} x k \cosh \psi) \cosh \psi / 4 \mathrm{~d} \psi}{\cosh \psi / 2-\sin \alpha / 2} .
\end{aligned}
$$

From (9.4) we observe that $\phi(x, 0), x \rightarrow-\infty$ is asymptotic to $\exp \left(\mathrm{i} x k_{x}\right.$ ) plus a term of the form $\exp (-\mathrm{i} x k) / \sqrt{-x}$. 
For $x>0$, we first examine $\phi(x,-0)$. Now the path $\mathscr{L}$ is closed in the upper half plane and there is a pole at $w=k \cos \alpha$ as well as a branch cut from $k$. If we now deform the path about the branch cut and take into account the pole we get $\phi(x,-0)=0$, since the integrals along the branch cut combine and vanish and the residue term cancels the term $\exp \left(i x k_{x}\right)$. For the examination of $\phi(x,+0)$, we find that the residue term still cancels and the integrals along the branch cut from $k$ double, thus verifying that the left side of (9.4) reduces to the right side. Similar comments can be made about $\hat{F}_{1}(w)$ and $\hat{F}_{2}(w)$.

\section{References}

[1] Bart, H.; Gohberg, I.; Kaashoek, M. A.: Operator Polynomials as Inverses of Characteristic Functions. Integral Equations Operator Theory 1 (1978) 1-18

Bart, H.; Gohberg, I.; Kaashoek, M. A.: Minimal Factorization of Matrix and Operator Functions. In: Operator Theory: Advances and Applications. Basel: Birkhauser 1979, 227 p.

[2] Carleman, T.: Sur La Résolution De Certaines Equations Intégrale. Arkiv Fơr Mat., Astr. Och Fysik 16 (1922) $1-19$

[3] Carlson, J. F.; Heins, A. E.: The Reflection of an Electromagnetic Plane Wave by an Infinite Set of Plates I. Quart. Appl. Math. 4 (1947) 313 - 329

Heins, A. E.; Carlson, J. F.: The Reflection of an Electromagnetic Plane Wave by an Infinite Set of Plates II. Quart. Appl. Math. 5 (1947) $82-88$

Heins, A. E.: The Reflection of an Electromagnetic Plane Wave by an Infinite Set of Plates III. Quart. Appl. Math. 8 (1950) 281 - 291

Heins, A. E.: The Green's Function for Periodic Structures in Diffraction Theory With an Application to Parallel Plate Media, I and II. J. Math. Mech. 6 (1957) 401-426; 629-639

[4] Elliott, J.: On Some Singular Integral Equations of the Cauchy Type. Ann. of Math. 54 (1951) $349-370$

[5] Heins, A. E.: Systems of Wiener-Hopf Integral Equations and Their Application to Some Boundary Value Problems in Electromagnetic Theory. Proc. Symp. Appl. Math., Amer. Math. Soc., Vol. 2 (1950) 76-81

[6] Hurd, R. A.: The Wiener-Hopf-Hilbert Method for Diffraction Problems. Canadian. J. Phys. 54 (1976) $775-780$

[7] Levine, H.; Schwinger, J. S.: On the Radiation of Sound from an Unflanged Circular Pipe. Phys. Rev. (2) 73 (1948) $383-406$

[8] Paley, R. E. A. C.; Wiener, N.: Fourier Transforms in the Complex Domain. Amer. Math. Soc. Collog. Publ. XIX (1934)

[9] Rawlins, A. D.: The Solution of a Mixed Boundary Value Problem in the Theory of Diffraction by a Semi-Infinite Plane. Proc. R. Soc. Lond. A 346 (1975) $469-484$

[10] Sommerfeld, A.: Mathematische Theorie der Diffraction. Math. Ann. 47 (1896) 317-374

[11] Tricomi, F. G.: Integral Equations. New York 1957: Interscience Publ.

Professor Albert E. Heins

Department of Mathematics

University of Michigan

Ann Arbor, MI 48109, USA

(Received January 28, 1981) 